\title{
Development and Application of Sheet-like Flexible Carbon Fiber Heat-generating Element
}

Tao Ma

State Grid Liaoning Energy Saving Services Co. Ltd

Shenyang, China

e-mail:mataosy@163.com

Ning Wang

State Grid Liaoning Electric Power Supply Co. Ltd Shenyang, China

e-mail: wangningsy@163.com

\author{
Lina Qi \\ Anshan Guangyuan Tech. Products Co. Ltd \\ Anshan, China \\ e-mail: qilinaas@163.com
}

Ru Jia

Anshan Institute of New Material Application \&

Development

Anshan, China

e-mail: jiaruas@163.com

\begin{abstract}
This paper studies the carbon fiber structure, physical properties, chemical properties as well as classification of the carbon fibers and the application of carbon fiber, through appropriate material selection (the merits of the material), a large number of experiments (of manufacturing a heating element) and an accurate analysis of the experimental results, we have successfully combine the electrical properties of carbon fiber and soft performance of insulation materials, developed a body which can be used for people to make a variety of heating, insulation clothes, gloves, knee pads and insoles and other products, some products just over a year sales and marketing, and achieved good economic and social benefits. Practice has proved, the product have good heat insulation properties of the product, the product technology is advanced, mature production technology can be widely used in all aspects of production and life, upon retrieval of the item product technology for domestic initiative.
\end{abstract}

Keywords- structure; properties; experiments; benefits; practice

\section{INTRODUCTION}

In today's rapid social development, the environment, air, Pm2.5 and other phrases that people are very concerned about the current problem, and protect the body from harmful environmental and cold weather, clothing clothes as one of the important function of a clear understanding of the people the total area of $1.5-2.0 \mathrm{~m}$ about skin is the body most vulnerable to the weak parts, under normal circumstances and the general working conditions of ordinary people wearing protective clothing will be able to achieve its purpose, but under special operating environment, such as remote radar station for cold regions, pastoral, post and telecommunications utilities, especially electricity in winter in field operations staff, etc., must adhere to under cold harsh environment, the importance of the completion of a variety of rich, boring but uninterrupted duplication of work, in this personnel working under the environment are susceptible to various diseases: stomach, rheumatism, skin, frostbite and other occupational diseases. Therefore, the research and development of flexible planar carbon fiber heating element is very important, this article from the use of the electrical properties of carbon fiber and soft performance of insulation materials combine successfully developed, people can develop a variety of heat produced for human , holding clothes, gloves, knee pads and insoles and other products, some products are only marketed more than a year, and achieved good economic and social benefits [1].

Composite conductive polymer composite material is a composite material of polymer matrix, filler, carbon black, graphite, metal particles, inorganic salts, metal fibers, carbon fibers prepared from conductive particles. This class has both a conductive material, but also has many excellent properties of polymers, thus shielding electronic equipment and instruments and anti-electromagnetic interference, field battery electrode materials, antistatic materials, as well as a positive temperature coefficient (PTC) materials have been wide range of applications. Electrically conductive carbon fiber as a fibrous filler, using a conventional processing method of a composite conductive polymer composite material, such as blending, extruding, coating, lamination or the like, damage to the carbon fiber length is large, the dispersion uniformity of the carbon fiber in which poor thus affecting the conductive polymer composite materials resistance uniformity. In this paper processing methods, a new carbon fiber conductive composites, the upcoming chopped conductive carbon fibers with special chemical and physical surface treatment, and pulp fibers or chemical fibers mixed pulp, using conventional papermaking process to produce carbon fiber conductive composite paper, then with an insulating material, an electrode formed by lamination, etc. to form a new carbon-fiber laminates of the planar heat generating material. In this paper the influence of the kind of the pressing process of the planar heat generating material on its electrical properties and other properties such as resistance stability, long-term stability of the power, the far-infrared radiation characteristics, etc.

Practice has proved, good heat insulation properties of the product, the product technology is advanced, mature 
production technology can be widely used in all aspects of production and life, upon retrieval of the item product technology for domestic initiative [2].

\section{SELECT MATERIAL}

As we all know carbon fiber material is a metal and non-metallic materials in electrical and thermal conductivity of the best good conductor, therefore, like polypropylene Mostly clear based carbon fiber, pitchbased carbon fiber, viscose-based carbon fibers are different varieties of modern high-tech carbon fiber heating materials the matrix material, in actual use, what kind of carbon fiber or carbon fiber hybrid type, are based on the specific product requirements depending on the situation [3].

Carbon fiber is a carbon content of at least $95 \%$ of the high strength, high modulus fiber material of new fibers. It is composed of organic fiber such as flake graphite crystallites along the fiber axis direction of the pile made of the graphite crystallites and the carbonized material obtained by the graphitization treatment. Carbon fiber "in a velvet glove", quality lighter than aluminum, but the intensity is higher than steel and corrosion resistant, high modulus characteristics, in terms of military and civilian defense are important materials. It not only has the inherent intrinsic properties of carbon materials, and both soft and workability of textile fibers, a new generation of reinforcing fibers [4].

Carbon fiber has many excellent properties, the axial strength and high modulus carbon fiber, low-density, highperformance ratio, no creep under a non-oxidizing environment, high-temperature resistant, good fatigue resistance, and specific heat conductivity between the nonmetallic and between the metal, and has a low coefficient of thermal expansion anisotropy, good corrosion resistance, $\mathrm{X}$-ray transmission resistance. Good thermal conductivity properties, electromagnetic shielding and good.

\section{A. Composition structure}

Carbon fiber is a carbon content higher than $90 \%$ of the inorganic polymer fibers. Where in said graphite fibers of the carbon content higher than 99\%. Microstructure similar to carbon fiber artificial Graphite, is turbo static structure. Spacing between all levels of carbon fibers is about 3.39 to $3.42 \mathrm{~A}$, the level of individual carbon atoms in each of the parallel between the graphite as good as regular arrange, between the layers connected together by van per Waals forces.

\section{B. Material properties}

Carbon fiber carbon material both strong and soft fiber tensile workability two features, is one excellent mechanical properties of the new material. Carbon fiber tensile strength is about 2 to $7 \mathrm{GPa}$, tensile modulus of about 200 to $700 \mathrm{GPa}$. Density of about 1.5 to 2.0 grams per cubic centimeter, which is in addition to the original structure of silk, mainly determines the temperature in the carbonization process. Usually after $3000{ }^{\circ} \mathrm{C}$ high temperature treatment of graphite, the density of up to 2.0 grams per cubic $\mathrm{cm}$. Plus it's very light weight, its specific gravity lighter than aluminum, quarter, compared with the strength of steel is 20 times less than iron. The thermal expansion coefficient of the carbon fibers with other fibers is different, it has anisotropic characteristics. Carbon fiber is the specific heat capacity is generally 7.12. The thermal conductivity decreases with increasing temperature, the fiber direction is parallel to the negative ( 0.72 to 0.90$)$, and perpendicular to the fiber direction is a positive value (32 to 22). A specific type of carbon fiber and fiber-related resistance is at $25{ }^{\circ} \mathrm{C}, 775$ high modulus, and high-strength carbon fibers per $\mathrm{cm} 1500$. This allows the carbon fiber having the highest specific strength and specific modulus in all high-performance fibers Compared with titanium, steel, aluminum and other metal materials, the physical properties of carbon fiber has a high strength, high modulus, low density, linear expansion coefficient, etc., can be called the king of the new material [5].

The chemical properties of carbon fiber and the carbon met, it can be a strong oxidizing agent in addition to, the general basic inert. When the air temperature is higher than $400{ }^{\circ} \mathrm{C}$ apparent oxidized to produce $\mathrm{CO}$ and $\mathrm{CO} 2$. Carbon fiber on common organic solvents, acids, alkalis have good corrosion resistance, insoluble swelling, outstanding corrosion resistance, totally rust problem does not exist. Some scholars in 1981 the PAN-based carbon fiber soaked in alkali sodium hydroxide solution, the time has past 30 years, it remains the fiber morphology. But poor impact resistance, easy to damage, oxidation occurs under strong acid, an electromotive force is positive carbon fibers, and aluminum alloy for the negative electromotive force. When the carbon fiber composite materials, metal carbide and aluminum are composite applications, carburizing and electrochemical corrosion will occur. Therefore, the carbon fibers subjected to surface treatment before use. Carbon fiber is as well as oil, anti-radiation, anti-radiation, neutron absorption of toxic gases and deceleration characteristics.

\section{Classification}

Carbon fiber according to sources of raw materials can be divided into polyacrylonitrile-based carbon fiber rayon-based carbon fiber, phenolic-based carbon fiber, vapor-grown carbon fibers; press performance can be divided into general-purpose, high strength, medium modulus and high strength, high model and super model carbon fiber; Press state is divided into filaments, staple fibers and chopped fibers; divided by the mechanical properties and high-performance general-purpose type. General-purpose carbon fiber strength of $1000 \mathrm{MPa}$, a modulus of about $100 \mathrm{G} \mathrm{Pa}$. High-performance carbon fibers are divided into high-strength (strength of $2000 \mathrm{MPa}$, modulus 250G Pa) and high model (modulus 300G Pa above). Strength greater than $4000 \mathrm{MPa}$, also known as ultra high strength type; modulus greater than $450 \mathrm{G} \mathrm{Pa}$ called ultra-high model. With the development of the aerospace and aviation industry, and also appeared in carbon fiber high strength and high elongation, elongation greater than $2 \%$. The largest amount of polyacrylonitrile PAN-based carbon fibers is on the market more than $90 \%$ carbon fiber to PAN-based carbon fiber-based. Because 
carbon fiber veil of mystery has not been completely lifted, people still can not directly use carbon or graphite to make, but to use some of the carbon-containing organic fibers (such as nylon, acrylic, rayon, etc.) as raw materials, organic fibers and plastic resins combined with carbon fibers obtained by carbonization [6].

\section{EXPERIMENTAL PART}

\section{A. Raw materials}

Carbon fiber conductive paper width of $500 \mathrm{~mm}$ (Anshan Guang Yuan tech products Co., Ltd.), weight 50g / $\mathrm{m} 2$.

Mix pieces (Anshan Guang Yuan tech products Co., Ltd.): Thickness $1.0 \mathrm{~mm}-2.0 \mathrm{~mm}$ the main component of natural rubber, silicone rubber.

Strengthen the skeleton and the electrode (Anshan Guang Yuan tech products Co., Ltd.).

\section{B. Sample Preparation}

According to $(350 \mathrm{~mm} \times 350 \mathrm{~mm}$ sheet cutting mix); (300mmx300 $\mathrm{mm}$ cut conductive sheet, the electrode width of $15 \mathrm{~mm}$, the release film in the mold cavity by, mix sheet reinforcing skeleton layer, a conductive sheet, the electrode, enhancing skeleton layer, kneaded film, release film laminations, placed $400 \times 400 \mathrm{~mm}$ plate vulcanizing press, the curing process: at $150{ }^{\circ} \mathrm{C}$, 5Mpa under vulcanization 10 minutes, holding pressure and cooled to obtain rubber / carbon fiber composite conductive heating plate [7].

\section{Analysis of the test}

Cconductive paper resistance: self-made jig in the sample at the pressure across the electrodes, the electrode width is $20 \mathrm{~mm}$, the volume resistivity measured.

The heating plate resistance: direct measurement with a digital millimeter.

Resistance - temperature characteristics: the heating plate was placed in an oven heated by a certain heating rate, constant resistance was recorded after 5 minutes at a temperature determined.

V characteristics: the current measured at the beginning and a stable value of different input voltages.

Relationship between the power density and the surface temperature of: at different input power, the measured surface of the heating plate 20 evenly distributed points of the surface temperature, the mean value.

Operating is under long-term power stability resistance: set under power density $0.1 \mathrm{~W} / \mathrm{cm} 2$, applying a certain voltage, power running, recording resistance under different time.

\section{The heating element functions}

A planar heating element made of the above materials and technology, the network circuit resistance is relatively large, and thus the voltage applied to the heating after the formation of a stable heat generation. Its surface temperature is a linear relationship between the applied voltage, can be seen from below, when the applied voltage is $36 \mathrm{~V}$, the surface temperature can be about $80^{\circ} \mathrm{C}$. We joined in the mass production of insulation voltage of $12 \mathrm{~V}$ gloves, knee pads, and insulation clothes, (electric power supply can be washed lithium battery) only one minute flu Warm, three minutes after the surface temperature will stabilize at $35-40{ }^{\circ} \mathrm{C}$, after one hour is not hot, Just slightly above body temperature, if you wear the gloves in the winter motorcycle Mercedes-Benz in the field or on the insulation of clothing worn in field operations will not feel cold.

\section{E. Techniques}

To get good quality carbon fiber, you need to pay attention to technical points:

Achieve a highly purified raw silk, high strengthen, densification and flawless smooth surface is the primary task of preparing high-performance carbon fibers. Carbon fiber systems engineering need to start from the polymerization of monomer raw silk. Determine the quality of both the precursor of carbon fiber properties, but also its production cost constraints. PAN precursor is the most important quality a prerequisite for the manufacture of high-performance carbon fibers [8].

Impurity defect minimization is to improve the tensile strength of carbon fiber fundamental measures, but also a hot topic of research scientists. In a sense, the process of improving the strength is substantially reduced the process of decreasing defects.

In the pre-oxidation process, the premise of ensuring the homogeneous, pre-oxidation time is as short as possible. This is to reduce the production cost of the directivity subject.

Study of high temperature and high temperature equipment and related technology an important component. High-temperature carbonization temperature is generally at 1300 to $1800{ }^{\circ} \mathrm{C}$, graphitization generally between 2500 $3000{ }^{\circ} \mathrm{C}$. Under such a high temperature operation, both to run continuously, but also improve the life of the device, so a new generation of high-temperature technology research and high temperature equipment is particularly important. Microwave is under inert gas protection, such as, the oxygen-free state, plasma and induction heating techniques [9].

\section{APPLICATIONS}

Protection of human aspects: can be made heating gloves, vests, belts, knee pads, boots, and mattresses. No heating equipment for indoor heating in cold regions.

Industry: for a large tank, tank and vessel insulation materials, material storage and transportation pipelines, as well as insulation fermented or heated brewing industry source.

Agriculture: for greenhouses, greenhouse breeding, nursery and feeding of poultry heat.

Other areas: radar communications anti-icing alpine region, frost materials, cold wild frontier post and telecommunications utilities operating personnel, construction temporary field camp to take the warm source, and marine ship deck antifreeze insulation materials[10].

In addition, it can be used in high-temperature furnace insulation materials (the need to protect in an inert atmosphere). In today's turn of the century, humans may get involved in the survival of the natural or human 
environment and the surrounding climate becomes more severe. For scientific development and social progress needs to match, had only played naked people require clothes and other items, gives it a fire-retardant, non-toxic, anti-fog, insulation, thermal insulation, electrical conductivity, insulation, etc., it is set to wear resistance, aesthetics, comfort and economic practicality multifunctional high-tech products, it will be the advent of a new energy materials revolution.

Given the product technology is mature, stable performance, fast to its series of deep processing products to the market, will achieve good economic and social benefits.

\section{CONCLUSION}

In this paper, we introduce the carbon fiber structure, physical properties, chemical properties as well as classification of the carbon fibers and the application of carbon fiber, through appropriate material selection (the merits of the material), a large number of experiments (of manufacturing a heating element) and an accurate analysis of the experimental results, we have successfully combine the electrical properties of carbon fiber and soft performance of insulation materials, developed a body which can be used for people to make a variety of heating, insulation clothes, gloves, knee pads and insoles and other products, some products just over a year sales marketing, and achieved good economic and social benefits. Practice has proved, the product have good heat insulation properties of the product, the product technology is advanced, mature production technology can be widely used in all aspects of production and life, upon retrieval of the item product technology for domestic initiative.

\section{ACKNOWLEDGMENT}

The authors gratefully acknowledge the contribution of co-workers and reviewers' comments.

\section{REFERENCES}

[1] J.M.Zhang, "after finishing the comprehensive development to bring to market demand technology," Wool Technology,vol. A47,pp. 55-62, April 2000

[2] F.H. "Electric properties of carbon fiber and its application," New carbon Materials, vol.41,pp.14-19, May 1995

[3] H.Lian,X.D.Zhao, and D.S.Zhang,"Research on safety management of construction project in lean," Jou.Changsha.Dis.Voc.Tech.Co, vol. 2,pp.62-64,May 2010

[4] H.S.Zheng and S.R.Zhu, "epoxy matrix and carbon fiber monofilament Temperature-sensitive effect of its interface," Functional materials, vol.8,pp. 2079-2082, May 2012

[5] Y.X.Guo, "Modification mechanism of the surface- treated PANbased carbon fiber by electrochemical oxidation," Jour. Inorg.Mat, vol.24,pp.853-858, July 2009

[6] J.Zhu, "Effect of moisture absorption on damping and dynamic stiffness of carbon fiber/epoxy compos-ites," Jou.Mec.Scie. Tech,vol.23,pp.46-48,August 2009

[7] B.Wang,J.J.Jiang,C.Q.Kang , and Q.Xia,"The main idea of design standard of American power market and the design of electric power market of China,"Pow.Sys.Tech,vol.3,pp.24-27, July 2004

[8] L.J.Xu, "Promoting the fine management significance of power supply enterprise," Sci.Tech.Inform.Dev.Eco.vol.14,pp.24-27, May 2010

[9] M.Zeng and G.Tian, "The analysis and design of the wholesale electricity market of Latin American countries and Its Enlightenment to China ," China .Elec. Pow.News, vol.18 ,pp.8287, April 2009

[10] S.Pillay and U.K.Vaidya "Effects of moisture and UV exposure on liquid molded carbon fabric reinforced nylon 6 com-posite laminates," Com.Sci.Tech. vol.69,pp.839-846, April 2009 